\title{
Diffusion treatment of quantum theory and gravity. 3. Cosmology of diffusion gravity
}

\begin{abstract}
Zahid Zakir
Abstract

The diffusion treatment of quantum mechanics and gravity described in the previous two papers was based on the fact of the existence of a background field whose energy density determines the rate of quantum fluctuations, i.e. the rate of proper times, and gravity is a local deficit of this energy density. In cosmology, due to the conservation of the background field energy during the expansion of space, the energy density of this field decreases both locally and globally. A change in the distribution of the local energy density deficit of the background field over time leads to a deformation of the gravitational potential of galaxies and clusters, which can explain the plateau in the rotation curves, as well as the relation of masses to rotation velocities and velocity dispersions. A global decrease in the background energy density during expansion leads to cosmology with slowing down proper times. In earlier epochs, fluctuations in the background field were faster and the photon frequencies were greater than current ones. As a result, a third mechanism is added to the two mechanisms of frequency shift, the Doppler effect and the stretching of wavelengths - the violetshift at emitting in the early epochs. This shift compensates the redshift due to stretching, and ultimately only the redshift from the Doppler effect is observed, as well as the relativistic aberration for apparent luminosity. The basic relationships of the model of relativistic cosmology with a slowing down time are presented, including the "distance modulus - redshift" relation. It is shown that the model solves the main cosmological problems without new hypotheses and describes observations without dark matter and dark energy. Some changes in the picture of the evolution in early epochs are discussed, including changes in the properties of CMB.
\end{abstract}

Keywords: cosmological expansion, galaxy rotation curves, dark matter, modification of Newton's potential, cosmological redshift, cosmological models, early universe, $C M B$

\section{Content}

Introduction

1. Deformation of the field of galaxies and clusters during expansion....................................3

1.1. Rotation curves of galaxies from deformation of potential during expansion ................. 3

1.2. Relations between mass and rotation speed of galaxies and clusters ............................6

2. Three mechanisms of the cosmological frequency shift .......................................................7

2.1. Two redshift mechanisms: Doppler effect and stretching ......................................... 7

2.2. Third mechanism: violetshift at emission and observed Doppler shift ........................ 10

3. Relativistic aberration in cosmology ..............................................................................12

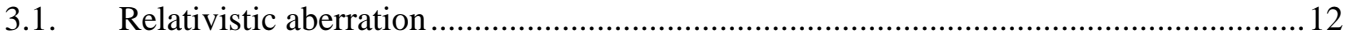

3.2. Aberrational fading of the apparent luminosity of distant objects ............................... 13

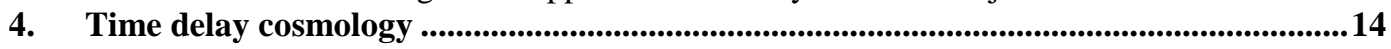

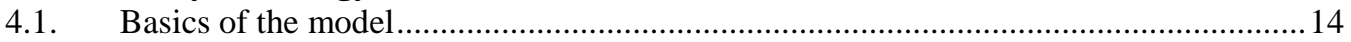

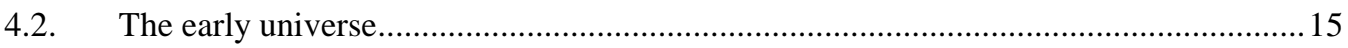

5. Observable effects of the time delay cosmology .............................................................17

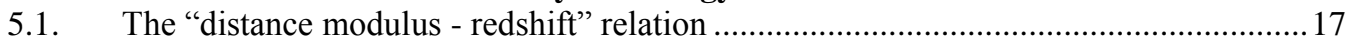

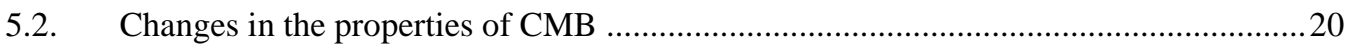

5.3. The lack of the former cosmological problems in the model......................................22

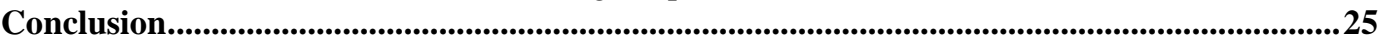

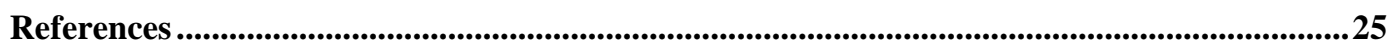

${ }^{1}$ Center for Theoretical Physics and Astrophysics, Tashkent Uzbekistan, zzakir@ qgph.org 


\section{Introduction}

The diffusion quantum mechanics, formulated in the first of papers [1], led to the diffusion gravity described in the second of papers [1]. In this paper, the main changes in cosmology following from the diffusion treatment of gravity will be studied.

One of the main consequences of the treatment of gravity as a local energy deficit of the background field is the variability in time of the magnitude and distribution in space of this deficit during the expansion of space. This leads to deformation of the gravitational potential of galaxies and their clusters, which is why it is proposed to explain unusual rotation curves, as well as the relations of the masses of galaxies with their rotation speeds or velocity dispersions. Then the same relationships for clusters can be explained similarly.

Cosmology deals with not only by a local, but also a global decrease in the energy density of the background field in the expanding space. In earlier epochs, respectively, this density was greater, fluctuations should have been more intense, and the photon frequencies should have been greater than current ones.

As a result, in addition to the two well-known mechanisms of cosmological frequency shift, the Doppler effect and stretching, there appears a third mechanism. This is the violetshift of photon's frequency at emission in earlier epochs. This violetshift is then compensated by the redshift due to the wavelength stretching along the path, and therefore the observed shift will be only the redshift due to the Doppler effect. For apparent luminosity, relativistic aberration must also be taken into account.

In general case, the diffusion gravity leads to cosmology with local proper times slowing down during expansion. The basic relationships of the corresponding model of relativistic cosmology are presented below. The modified "distance modulus - redshift" relation, taking into account the slowing down of local times in later epochs, describes the observations without hypotheses about the dark energy and dark matter.

It is shown that the new model solves the main cosmological problems naturally and without new hypotheses. These are the problems of horizon, homogeneity, flatness, cosmological constant, entropy, dark matter and initial singularity. The problems of horizon and homogeneity are explained by the fact that in the early epochs the light speed was greeter and, respectively, the radius of the horizon increased much faster than as estimated by using the current value of light speed.

The cosmology of diffusion gravity is presented in more details in the book [2].

In Section 1, the deformation of the field of galaxies and their clusters during expansion are studied. In Section 2 three mechanisms of cosmological frequency shift are considered. In Section 3 the need for accounting of the relativistic aberration in cosmology is shown. In Section 4 a model of cosmology with slowing down time in later epochs is formulated. The observable consequences of the model, including the description of redshifts and the solution of the cosmological problems are discussed in Section 5. 


\section{Deformation of the field of galaxies and clusters during expansion}

\subsection{Rotation curves of galaxies from deformation of potential during expansion}

The Newtonian gravitational potential has so far been extrapolated to galaxies and their clusters. For a galaxy of the baryon mass $M$ the equilibrium of the centrifugal and gravitational forces then gives a rotation curve:

$$
\frac{v_{c}^{2}}{r} \simeq \frac{G M}{r^{2}}, \quad v_{c}^{2}(r) \simeq \frac{G M}{r} .
$$

Observations showed that spiral galaxies are characterized by a plateau in their rotation curves, on average, approximately close to a constant velocity $v_{c}(r) \sim$ const.

To explain this fact, without abandoning the Newtonian potential, the hypothesis of the presence of a non-baryonic dark matter in galaxies was accepted. However, this hypothesis led to a number of problems [4].

First, the fraction of dark matter in total mass must be many times greater than the fraction of ordinary matter. Secondly, it should gravitate, but be distributed inversely than gravitating matter should be distributed, i.e. artificial dependence $M_{t o t}(r) \sim M_{0} r$ is required. Thirdly, dark matter has not yet manifested itself in any of the numerous experiments and observations. Fourthly, no traces of this dark matter have yet been found inside our galaxy. All this is so strange, inexplicable and even irrational that it makes fantastic this hypothesis based only on the ambition to preserve Newton's potential.

In diffusion gravitation, such rotation curves of galaxies appear naturally due to deformation of the energy density of the background field during the cosmological expansion of space and there is no need for dark matter.

Since the formation of stars and their clusters, it has dominated attraction in their vicinity, not the cosmological expansion and, therefore, their potentials have remained Newtonian. However, for galaxies and their clusters this is no longer the case. In the early epochs, when the expansion speed was greater than the current one, the radius $r_{c}$ of the gravitationally bound region (GBR) was several times smaller than the current one, so that the vicinity of galaxies and their clusters was in the expansion region. As a result, a part of their initial Newtonian potential $\varphi(r)$ that was in the expansion region for some time, was deformed due to the conservation of the background field energy in each of the expanding layers.

Let us evaluate the nature and degree of this deformation of the potential of galaxies [4]. Suppose that in the early epoch $t_{0}$, when neighboring galaxies began to move away from each other, a certain spatial layer around the galaxy with an initial radius $r_{0}$ and thickness $\Delta r_{0}$ began to expand. At a later time $t$, the radius and thickness of this layer will increase $r_{0} \rightarrow r$, $\Delta r_{0} \rightarrow \Delta r$, moreover, in approximately the same proportion:

$$
\frac{\Delta r}{\Delta r_{0}} \simeq \frac{r}{r_{0}}, \quad \Delta r \simeq \frac{r}{r_{0}} \Delta r_{0} .
$$

Let us now consider the effect of this fact on the gravitational potential in this layer.

Suppose that at the initial moment the deficit of the background field energy density $W(r)$ in a layer $\Delta r=r_{2}-r_{1}$ was proportional to the difference of a potential $\varphi(r)$ : 


$$
U\left(r_{2}, r_{1}\right)=-\int_{r_{1}}^{r_{2}} \frac{(\nabla \varphi)^{2}}{8 \pi G} d V=\left[\varphi\left(r_{1}\right)-\varphi\left(r_{2}\right)\right] M / 2 .
$$

At the expansion of space, the total energy of the background field in this layer should be conserved despite the fact that the layer moves away from the galaxy and its width increases. This means that the energy levels in this background field "decrease" and the corresponding "energy deficit" increases. But this, on the other hand, means deepening that potential well, which forms the gravitational potential. Therefore, this leads to an effective increasing the gravity of sources during cosmological expansion.

However, such a decrease occurs everywhere, since the expansion is global, and therefore more important is how this will affect the potential difference at the boundaries of the expanding layer. Since the values of the gravitational potential at these boundaries decrease equally during expansion, the potential difference between these boundaries remains unchanged. This means that values of the potential are comoving to expansion. Thus, in expanding space, the fields of galaxies correspond to comoving potentials. Since, according to (3), the potential difference $\phi\left(r, r_{0}\right)$ determines the energy of the gravitational field in the layer, this also means the conservation of the gravitational energy in this layer during expansion.

The expansion speed $H=\dot{a} / a$, where $a(t)$ is the scale factor of cosmological expansion, decreases in time, and since the radius of GBR is defined as $r_{c}(t) \sim H(t)^{-2 / 3}$, it increases with time $r_{c}(t)>r_{c}\left(t_{0}\right)$. In the layer with then initial width $\Delta r_{0}$, that has expanded and become larger, the conditions for the energy conservation (3) and the equality of the potential difference take the form:

$$
\begin{gathered}
U\left(r_{0}+\Delta r_{0}, r_{0}\right)=U(r+\Delta r, r), \\
\varphi\left(r_{0}+\Delta r_{0}\right)-\varphi\left(r_{0}\right)=\phi\left(r+\Delta r, r_{0}\right)-\phi\left(r, r_{0}\right) .
\end{gathered}
$$

Let initially a potential of the layer $r_{0}$ was Newtonian with acceleration $\sim 1 / r_{0}^{2}$, and after expansion it became a comoving potential with a modified distance dependence of acceleration $\sim 1 / f(r)$. Then, to find $f(r)$, we can write the condition (5) in the form:

$$
-\frac{G M}{r_{0}^{2}} \Delta r_{0}=-\frac{G M}{f(r)} \Delta r=-\frac{G M}{f(r)} \cdot \frac{r}{r_{0}} \Delta r_{0},
$$

which gives $f(r)=r_{0} r$. As the result, we find the modified expression for the gravitational acceleration $w_{g}\left(r, r_{0}\right)$ :

$$
w_{c}\left(r, r_{0}\right)=-\frac{G M}{f(r)}=-\frac{G M}{r_{0} r} .
$$

Thus, the Newtonian potential after stretching during the expansion of space passes into the comoving potential $\varphi \rightarrow \phi$ and, accordingly, the gravitational acceleration is modified $w_{N}(r) \rightarrow w_{c}\left(r, r_{0}\right)$.

As the result of this fact, at the comoving potential with the acceleration $w_{c}\left(r, r_{0}\right)$, the balance of gravitational and centrifugal forces leads to the approximately constant rotation speed of the spiral galaxy in the region $r>r_{0}$ : 


$$
\frac{v_{0}^{2}(r)}{r}=\frac{G M}{r_{0} r}, \quad v_{0}^{2}(r)=\frac{G M}{r_{0}} \sim \text { const } .
$$

Notice that although the $r_{0}$ was of order the galaxy's size, the region of change $r\left(r_{0}\right)$ can be significantly larger. Thus, the formation of a plateau in the rotation curves of galaxies follows from (8) and $r_{0}$ can be determined from the masses and rotation speeds $r_{0}=G M / v_{0}^{2}$. As an effective value of $r_{0}$, one can take from one third to half the size of the galaxy, depending on the degree of concentration of the mass of the galaxy in the central region.

To apply the comoving potential to the evolution of the field of the galaxy, one must also take into account the existence of the evolving radius $r_{c}$ of the GBR of the galaxy, inside which the attraction field dominates, which becomes almost static.

This radius corresponds to the distance where the potential energy of the particle in the gravitational field of the galaxy compensates the kinetic energy of the recession velocity $v_{H}(r)=H r$. This condition gives:

$$
\frac{1}{2} H^{2} r_{c}^{2}=\frac{G M}{r_{c}}, \quad r_{c}(t)=\frac{(2 G M)^{1 / 3}}{H^{2 / 3}(t)}
$$

Taking $H=h \times 100 \kappa M \times c^{-1} \times M p c$, with the modern values $h=h_{0}=0.7$ and masses $\quad M=\left(10^{10} ; 10^{11} ; 10^{12} ; 10^{13}\right) M_{\odot}$, we have $r_{c(0)}=(0.26 ; 0.56 ; 1.2 ; 2.6) M p c$, whereas in the early epochs with $h=10$ we have $r_{c(0)}=(0.044 ; 0.095 ; 0.20 ; 0.44) M p c$. Thus, during the formation of a galaxy, $r_{c}$ was of the order of its size and then increased with time as $r_{c} \sim H^{-2 / 3}$ due to a decrease of $H$ with growth of $a(t)$.

In the transition region, which expanded, and then appeared inside the GBR of radius $r_{c}(t)$, the gravitational potential will remain deformed. But now this is not a comoving potential, since the particles no longer participate in the expansion, but a stretched potential with the distance dependence (7). Integration of (7) leads to a logarithmic dependence for the deformed part of the potential. Adding the remainder of the Newtonian potential, we arrive at the stretched model potential:

$$
\phi \simeq-\frac{G M}{r}\left(p+q \frac{r}{r_{0}} \ln \frac{r}{r_{0}}\right), \quad r \geq r_{0} .
$$

Here, dimensionless coefficients $p, q$ express the specifics of the history of the expansion of each galaxy and can be selected from observations. The stretched potential decreases with a distance slower than Newtonian and expresses the effective amplification of gravity in the layers with a decrease in the "energy level" of the background field during expansion. 


\subsection{Relations between mass and rotation speed of galaxies and clusters}

From (9) we can also find a relationship between the rotation speed and the mass of the galaxy [4]. Suppose that, during the formation of galaxies, the density of baryonic matter was of the order of the density of baryonic matter in current galaxies $\rho_{0} \sim 10^{-24} \mathrm{~g} / \mathrm{sm}^{3}$.

For a homogeneous ball

$$
\rho_{0}=\frac{3 M}{4 \pi \bar{r}_{0}^{3}}, \quad r_{0} \simeq \frac{1}{3} \bar{r}_{0}=\left(\frac{M}{36 \pi \rho_{0}}\right)^{1 / 3}
$$

so from (9) it follows $v_{0}^{2} \simeq M^{2 / 3} \alpha_{S}^{-2 / 3}$ and for the connection of mass with the rotation speed we obtain:

$$
M \simeq \alpha_{S} v_{0}^{3}, \quad \alpha_{S}=\left(36 \pi \rho_{0} G^{3}\right)^{-1 / 2} .
$$

For a disk of thickness $d$ the density and $r_{0}$ are:

$$
\rho_{0}=\frac{M}{2 \pi \bar{r}_{0}^{2} d}, \quad r_{0} \simeq \frac{1}{3} \bar{r}_{0}=\left(\frac{M}{18 \pi \rho_{0} d}\right)^{1 / 2}
$$

Then from (9) it follows $v_{0}^{2} \simeq M^{1 / 2} \alpha_{d}^{-1 / 2}$ and:

$$
M \simeq \alpha_{d} v_{0}^{4}, \quad \alpha_{d}=\left(18 \pi G^{2} \rho_{0} d\right)^{-1} .
$$

Relations (11) and (14) give, in particular, a new estimate of the average density of galaxies:

$$
\rho_{0(S)}=\frac{3}{4 \pi G^{3} \alpha_{S}^{2}}, \quad \rho_{0(d)}=\frac{1}{2 \pi G^{2} \alpha_{d} d} .
$$

From observations, the empirical baryonic Tally-Fischer relation (BTF) for the rotation curves of spiral galaxies is known:

$$
M=\alpha v^{\beta}
$$

where $\alpha=$ const. and $\beta \simeq 3 \div 4$ in different estimates of the baryon mass $M$. These values of $\beta$ are consistent with model estimates for the ball $\beta_{S}=3$ and disk $\beta_{d}=4$.

For the average density of spiral galaxies $\rho_{0} \sim 10^{-24} \mathrm{~g} / \mathrm{sm}^{3}$, their thickness $d \sim 10 \mathrm{kpc}$, and the observed average values of the rotation speeds $v \sim 200 \mathrm{~km} / \mathrm{s}$, relations (12)-(14) give estimates for the parameter $\alpha_{s} \simeq 13, \alpha_{d} \simeq 65$, (in units $M_{\odot} \times s^{4} / \mathrm{km}^{4}$ ), which is close to the observed average value $\alpha \sim 50$ [ ].

For elliptical galaxies that do not rotate, the empirical Faber-Jackson relation $M=\alpha v_{d}^{4}$ for the velocity dispersion $v_{d}^{2}$ takes place. This ratio is also consistent with stretched potential.

Further, the results for the field of an individual galaxy can be applied to groups and clusters of galaxies. A plateau on the rotation curves also occurs in a system of two galaxies with a total mass $M_{1}+M_{2}$, which can be reduced to motion around the center of mass of an object with a reduced mass $\mu=M_{1} M_{2} /\left(M_{1}+M_{2}\right)$. Therefore, the stretching of the potential can also be checked by observing the dynamics of binary systems in which the rotation 
speeds, depending on the total mass, should be practically independent of the radius of the system.

If the size of the binary system of galaxies is close to the size of each of them, then part of the matter falls into the center of mass of the system, forming a bulge. In addition, the center of mass of each of the former galaxies with stretched potentials will rotate at the same speed, being the prototype of density waves, which later transform into two or more spiral arms.

A similar scenario can be realized in a system of three galaxies, where one of the galaxies can have direct or opposite speed, and the system can later be transformed into a spiral galaxy with three or more spiral arms. Thus, with a stretched potential, systems of elliptical or irregular galaxies can form spiral galaxies over time. In this case, the constancy of rotation curves and large angular momenta of spiral galaxies follow from the large angular momentum of the initial binary or triple system of galaxies.

Finally, considering the groups and clusters of galaxies within the framework of the new treatment, we find that the velocity dispersion in them will also be determined by the stretched potentials and relations (12) - (14). Since clusters are formed later than the epoch when the potential of galaxies formed and stretched due to the expansion, (12) - (14) give for the baryon mass of clusters the relation:

$$
\frac{M_{c l}}{M_{g a l}} \simeq \frac{v_{c(c l)}^{3 \div 4}}{v_{c(g a l)}^{3 \div 4}} .
$$

At $v_{c(g a l)} \sim 200 \mathrm{~km} / \mathrm{s}$ for $M_{g a l} \sim 10^{11} M_{\odot}$, in a cluster with a velocity dispersion $v_{c(c l)} \sim 1000 \mathrm{~km} / \mathrm{s}$, the formula yields $M_{c l} \sim 10^{14} M_{\odot}$, which is consistent with estimations from observations.

Thus, the comoving potential, which has turned into a stretched potential, decreases with distance more slowly than Newtonian potential and allows one to explain the main unusual astrophysics of galaxies attributed to the dark matter, and can be taken as a basic model in studies of structure formation.

\section{Three mechanisms of the cosmological frequency shift}

\subsection{Two redshift mechanisms: Doppler effect and stretching}

In the standard formulation of relativistic cosmology in the general relativity, depending on an authors' preference, one of the two mechanisms was specified as a source of redshift in the spectra of objects in the expanding universe, either the Doppler effect or the wavelength extension during propagation. The reason for this duality was that both explanations of the redshift were considered as two equivalent ways of describing the same phenomenon and, therefore, when one of them was taken into account, the other was completely ignored.

In reality, as will be shown below, they are two independent mechanisms of the appearance of cosmological redshift and must be taken into account together. The restriction only one of the effects was associated not with logical arguments, but with the desire to avoid doubling of the predicted redshifts, which would lead to a catastrophic disagreement between theory and observations.

It will be shown below that diffusion gravity also adds a third mechanism which compensates the contribution of one of the two former ones, leaving the contribution of only one of them, and thus leads to the agreement between the theory and observations. 
In flat static space, photons from a source moving away with the velocity $v(r)=H_{0} r$, where $H_{0}$ is a constant, will come to the observer at $r=0$ with a redshift $z_{D}$ due to the longitudinal Doppler effect. As a result, the observed wavelength $\lambda_{D}$ is related by the initial wavelength $\lambda_{0}$ by the formula:

$$
\frac{\lambda_{D}}{\lambda_{0}}=1+z_{D}=\sqrt{\frac{1+v / c}{1-v / c}}=\sqrt{\frac{1+H_{0} r / c}{1-H_{0} r / c}} .
$$

The distance up to the source is then expressed in terms of $z_{D}$ as:

$$
r=\frac{c}{H_{0}} \cdot \frac{z_{D}+z_{D}^{2} / 2}{1+z_{D}+z_{D}^{2} / 2} .
$$

In the first approximation (18) gives a linear growth of $z_{D}$ with distance $z_{D} \simeq H_{0} r / c$.

It is important here that this redshift already existed at the moment of emission of the photon, and then the photon propagated with this constant wavelength.

Now let the space is expanding and the frame of reference is static, i.e. non-comoving to expansion. The distance from the center to any object at the moment $t$ is equal to $r=a(t) \chi$, where $a(t)$ is the scale factor, and $v=H_{0} r$ is the expansion speed with $H_{0}=\dot{a}_{0} / a_{0}=$ const . If a source, resting in this static frame at a point $r=$ const. emits a photon, then the world line of this photon is given by:

$$
c^{2} d t^{2}-a^{2} d \chi^{2}=0, \quad c d t=a \cdot d \chi .
$$

During the propagation of the photon, the initial wavelength $\lambda_{0}$ increases in proportion to the growth of the scale factor $a_{0} / a$ and becomes equal to $\lambda_{E}$, related with $\lambda_{0}$ as:

$$
\frac{\lambda_{E}}{\lambda_{0}}=\frac{a_{0}}{a}=1+z_{E} .
$$

Thus, the photon will come to the center with the redshift $z_{E}$ due to the expansion of the space between the source and the detector. In the first approximation

$$
a \approx a_{0} \cdot\left(1+H_{0} \Delta t\right)=a_{0} \cdot\left(1+\frac{H_{0} r}{c}\right), \quad z_{E} \simeq \frac{H_{0}}{c} r \simeq \frac{v}{c},
$$

i.e. the linear growth occurs with a distance of both speed and $z_{E}$, which is similar to the longitudinal Doppler effect considered above.

Moreover, the source has a velocity $-H_{0} r$ with respect to the bodies comoving the expansion of space in its vicinity. There is no redshift immediately at emission of the photon, since the source is stationary relative to the center and therefore there is no Doppler effect, but in the future there is a wavelength extension due to expansion and redshift is accumulated during the photon's propagation.

Thus, we considered two mechanisms of the appearance of redshift in GR, when each of them appears in its pure form, and the second is absent. If in static space the source is resting in an expanding frame, then only the longitudinal Doppler effect is observed, which occurs at 
the moment of emission. If, on the contrary, space expands, and the source rests in the static frame, then only the wavelength extension during propagation is observed.

We now consider the third case, which is a combination of the two previous ones, and where, therefore, both mechanisms of redshift will appear together. This is the case when the space expands, but along with the static frame there is also a comoving frame expanding with the space.

Let a photon is emitted by a source at rest in the comoving frame, which means that the source moves by the speed $H_{0} r$ with respect to the static frame. Then at the emission, this photon will have a wavelength $\lambda_{D}$ with a redshift $z_{D}$ from (18) due to the longitudinal Doppler effect with respect to the photons emitted by another source resting in the static frame.

The wavelength $\lambda_{0}$ of the photon emitted in the static frame and the photon emitted from the comoving frame $\lambda_{D}$ are further stretched during their propagation proportionally $a$. At arriving to the observer, the wavelength of the first photon will become equal to $\lambda_{E}$ from (19), while the second photon with the initial wavelength $\lambda_{D}$ in the static frame will experience exactly the same stretching and at arriving will have the wavelength $\lambda_{D E}$, which we also find from (21):

$$
\frac{\lambda_{D E}}{\lambda_{D}}=\frac{a}{a_{0}}=1+z_{E} .
$$

Taking into account that according (18) it was $\lambda_{D}=\lambda_{0}\left(1+z_{D}\right)$ already in the beginning, we find the observed redshift $z$ as a combined redshift, expressed through $z_{D}$ and $z_{E}$ as:

$$
\frac{\lambda_{D E}}{\lambda_{0}}=\frac{\lambda_{D E}}{\lambda_{D}} \frac{\lambda_{D}}{\lambda_{0}}=\left(1+z_{E}\right)\left(1+z_{D}\right)=1+z .
$$

In the first approximation, this gives:

$$
z \simeq z_{E}+z_{D} \simeq 2 r \cdot H_{0} / c \simeq 2 v / c
$$

i.e. the contributions of the pure Doppler effect $z_{D} \simeq r \cdot H_{0} / c$ and pure stretch $z_{E} \simeq r \cdot H_{0} / c$ are summed and a double redshift should be observed than with one of the effects.

Since in reality a single effect is observed, then in the previous formulation of relativistic cosmology there was a catastrophic discrepancy between the theory and observations. This is the paradox of doubling of the cosmological redshift.

For this reason, one group of authors attributed the observed redshift to the Doppler effect, and the other group attributed the wavelength extension to the expansion of space. At the same time, both groups claimed that these are two ways of describing of the same phenomenon and that there is no contradiction. The fallacy of these intuitive statements is shown by the above thought experiments. Therefore, below we will consider this situation from the point of view of the diffusion gravity. 


\subsection{Third mechanism: violetshift at emission and observed Doppler shift}

In general relativity, two types of cosmological redshift, due to the Doppler effect and stretching, must be taken into account together. But then they contribute separately to the total redshift and the predicted redshifts will become twice as large as observed, which becomes a very serious problem.

If we continue to consider general relativity as correct on a cosmological scale, then the theory was apparently supplemented by some unrealistic assumption, which is allowed but not required by the theory.

In the framework of general relativity, which is an extremely general formalmathematical construction, it is difficult to find such a hidden assumption in the standard formulation of relativistic cosmology, which was shown by all previous experience in applying the theory. In contrary, diffusive gravity, being a more physical treatment, leaves no room for unrealistic assumptions and allows to find them.

Cosmology in terms of the diffusion gravity deals with a global decrease in the energy density of the background field during the expansion of space. This means that in earlier epochs, when the energy density of the background field was greater, the fluctuations of particles in this denser state of the background field should be more intense, and the frequency of photons should accordingly be higher than the current ones.

As a result, to the two mechanisms of cosmological shift, the Doppler effect and stretching, the third mechanism, the violetshift at emission of photons in earlier epochs, should be added. This violetshift then compensates the redshift due to the stretching of wavelengths at propagation. As a result, the observed shift appears as the Doppler redshift only.

Returning to the question of the hidden assumption mentioned above, we see that it consisted in the fact that in the former formulations, when a photon was emitted in the early epochs at a large distance, its wavelength was considered the same as it is now, at the time of reception, although there is no reason for this and this was not proved by anything.

In reality, in GR, the wavelength of a photon when it was emitted in an earlier epoch should coincide with the wavelength of the same photon at the place of registration only in the same early epoch. For example, the wavelength of a photon emitted far from us in the epoch of 10 billion years after the Big Bang in local time there should coincide with the wavelength of the same photon here in our epoch also in the epoch of 10 billion years after the Big Bang. This follows from the cosmological principle, based on the observed large-scale homogeneity of space and the distribution of matter in the same epoch, i.e. is not an intuitive statement, but a direct consequence of the basic principle of the theory.

After the comparison of the wavelength of the photon at the place of its emission and at the place of observation is carried out correctly, namely, in terms of the wavelength of the same photon near the observer in the same epoch, another problem arises - how is the wavelength of the photon of the observer in that epoch associated with the wavelength of the same photon in our epoch.

General relativity allows two opposite answers to this question, one of which, formally the simplest, was chosen purely intuitively. This answer lies in the fact that here the wavelengths of the same photons were the same in all epochs. In the frame comoving the expansion, the proper time was chosen as the time coordinate, the local rate of which remains unchanged. 


\section{Cosmology of diffusion gravity}

However, if we compare two photons, one of which came from far away, and its wavelength was stretched, and the second circulated for the same time here, then the absence of stretching of the "local" photon looks strange. After all, the cosmological principle requires the same cosmological effects everywhere, which means that stretching should act on all photons equally, wherever they propagate.

To justify the previous choice, one can cite the argument that the incoming photon was born long ago, and the local one, with which its wavelength is compared, is usually born in our epoch. In this regard, one should pay attention to the fact that the matter is not in the photons themselves, but in the energy levels of those atoms that emit them. But these levels depend on the local rate of flow of proper time. Therefore, for local photons, the question of their wavelength in different epochs reduces to the question of whether local proper time of atoms flows the same in different epochs.

If, as previously thought, it was assumed that the flow rate is the same in all epochs, then the above problem of doubling redshifts remains unsolved. Therefore, this assumption contradicts to the cosmological observations.

At the same time, the second assumption, also admissible in GR, that proper time flows in different epochs differently, leaves room for new effects that can solve this problem.

In this case, it is necessary to find out whether the rate of proper time speeds up or slows down during the expansion. Since the proper time is supposed as variable, it is necessary to choose a distinguished epoch, the time of which can be taken as the standard one and plays the role of the time coordinate. For us, as observers, our epoch is distinguished, since the signals from all previous epochs are compared with signals in our epoch. Then proper times $\tau$ are conveniently expressed in terms of our local proper time $t$ :

$$
d \tau^{2}=g_{00}[a(t)] d t^{2} .
$$

In general relativity, there remains arbitrariness in the choice of the time component of the metric $g_{00}$, since it can be either less than unity $g_{00}[a(t)]<1$ or more than it $g_{00}[a(t)]>1$ :

In diffusion gravitation, the choice turns out to be unambiguous - during the cosmological expansion, the rate of proper time slows down everywhere. This follows from the fact that during the expansion of space the energy of the background field is conserved and therefore its density decreases throughout the space. As a result, the intensity of quantum fluctuations of particles decreases everywhere, which means that the rate of processes, i.e. proper times are slowing down. The nature of this dependence is naturally the same as that of energies, i.e. the slowdown is proportional to the scale factor. For our proper times, therefore, we have:

$$
d \tau=\frac{a_{0}}{a} d t
$$

For the metric in $\left((26)\right.$, this means that $g_{00}(t)=a_{0}^{2} / a^{2}$, i.e. for earlier epochs with $a<a_{0}$ we have $g_{00}(t)>1$, but for future epochs with $a>a_{0}$ it will be $g_{00}(t)<1$.

Since in earlier epochs the rate of proper time was faster, then at each point the photon frequencies were greater, and the wavelengths were shorter than those of the same photons now. This leads to a violetshift in earlier epochs by a factor opposite to the stretching factor in (23). 
Therefore, if the wavelength of the photon during emission was to be equal $\lambda_{D}$ only when the Doppler effect was taken into account, then in fact the wavelength was shorter due to the initial violetshift. Denoting this wavelength as $\lambda_{C D}$, we can find it from the relation:

$$
\frac{\lambda_{C D}}{\lambda_{D}}=\frac{a}{a_{0}}=\frac{1}{1+z_{E}} .
$$

Then, the initial violetshift due to the going faster of time in (28) and the further redshift due to stretching from (23) should mutually cancel each other out and, as a result, the observed redshift should be reduced to the Doppler effect from (18). The observed wavelength $\lambda_{C D E}$, including the contribution of these three effects, is then calculated as the result of a chain of relations:

$$
1+z=\frac{\lambda_{C D E}}{\lambda_{0}}=\frac{\lambda_{C D E}}{\lambda_{D E}} \frac{\lambda_{D E}}{\lambda_{0}}=\frac{\lambda_{C D E}}{\lambda_{D E}} \frac{\lambda_{D E}}{\lambda_{D}} \frac{\lambda_{D}}{\lambda_{0}}=\frac{a}{a_{0}} \frac{a_{0}}{a} \frac{\lambda_{D}}{\lambda_{0}}=\frac{\lambda_{D}}{\lambda_{0}}=1+z_{D} .
$$

Thus, we really get the exact relations:

$$
1+z=\frac{\lambda_{C D E}}{\lambda_{0}}=\frac{\lambda_{D}}{\lambda_{0}}=1+z_{D}, \quad \lambda_{C D E}=\lambda_{D}, \quad z=z_{D} .
$$

In the first approximation, this gives a linear Doppler effect, which from the very beginning was considered an explanation of the observed linear dependence of $z$ on $r$ and $v$ :

$$
z=z_{D} \simeq r \cdot H_{0} / c \simeq v / c .
$$

Thus, in diffusion gravity, the paradox of doubling of the cosmological redshift even in the linear section, caused by the combined action of the Doppler effect and stretching during expansion, is solved naturally, since the initial violetshift compensates the redshift from stretching, leaving only the contribution of the Doppler effect.

\section{Relativistic aberration in cosmology}

\subsection{Relativistic aberration}

In classical physics, only the longitudinal Doppler effect takes place, which linearly depends on the velocity. The special relativity adds to the classical effect an even quadratic in speed relativistic effect, independent of the direction of the source velocity. Therefore, the relativistic effect in its pure form manifests itself in the transverse motion of the source, as the transverse Doppler effect, absent in classical physics.

Similarly, in classical physics there is only a linear in speed aberration of the light flux. The special relativity also adds a quadratic relativistic effect, independent of the direction of the source velocity.

It is important here that both the relativistic quadratic Doppler effect and the relativistic aberration are associated with the same relativistic factor $\left(1-v^{2} / c^{2}\right)^{1 / 2}$. Therefore, if there is a quadratic Doppler effect for the photon wavelength, then relativistic aberration for their flux will also take place.

In relativistic kinematics, the element of the solid angle $d \varphi d\left(\cos \theta^{\prime}\right)$ in the rest frame of the source differs from the same angle in the rest frame of the observer $d \varphi d(\cos \theta)$ due to the Lorentz transformation for $\cos \theta^{\prime}$ : 


$$
d(\cos \theta)=\frac{1-v^{2}}{\left(1+v \cos \theta^{\prime}\right)^{2}} d\left(\cos \theta^{\prime}\right) .
$$

For the flow from a receding source in the rest frame of the observer, $v \cos \theta^{\prime} \simeq-v$ and the observed element of the solid angle $\theta$ is found from:

$$
d(\cos \theta)=\frac{1+v}{1-v} d\left(\cos \theta^{\prime}\right)=\left(1+z_{D}\right)^{2} d\left(\cos \theta^{\prime}\right),
$$

where $\left(1+z_{D}\right)$ is the redshift factor due to the longitudinal Doppler effect.

As we see, the change in the solid angle depends on the same factor $\left(1+z_{D}\right)$ that determines the longitudinal Doppler effect and these two effects will contribute together - the first reduces the frequency of photons, and the second - the number of photons in a given solid angle. With an increase in the solid angle of the cone, the number of photons in the cone of the previous value decreases, and the apparent luminosity decreases. This relativistic decrease in the density of the light flux coming from receding objects must be taken into account in cosmology dealing with the expanding universe.

\subsection{Aberrational fading of the apparent luminosity of distant objects}

Observational cosmology deals with fluxes of photons from distant sources, which, due to cosmological expansion, move away from the observer at sufficiently high speeds. move away from the observer with speed $v(r)$ growing by the distance. Therefore, it is necessary to take into account relativistic aberration for the apparent luminosities of such sources.

The neglect of aberration in previous models of relativistic cosmology was related with the above-considered misconception that the cosmological redshift is supposedly caused only by stretching the photon wavelength. With this point of view, the Doppler effect was considered only as a clearer analogy to this purely geometric effect. And where there is essentially no Doppler effect, naturally there should not be an aberration. This was explained in geometric language by the fact that in the expanding space, solid angles are preserved in the light fluxes.

As was shown in the previous section, in the global static frame where the observer is resting, the Doppler effect takes place already at the beginning, immediately after the emission of the photon flux by a source that is moving away in this system. Therefore, there should initially be relativistic aberration, i.e. distortion of the solid angle of the flow and then this flow propagates in the expanding space already without changing the wavelength, as well as without changing the solid angle.

The apparent luminosity is inversely proportional to the solid angle of the initial flow and is defined as $l=L / 4 \pi d_{p}^{2}$, where $d_{p}$ is the photometric distance, $L$. Therefore, at taking into account of the aberration, the apparent luminosities of distant objects $\tilde{l}$ will be less than $l$. This leads to the effective increasing of the photometric distance $\tilde{d}_{p}$ with respect to $d_{p}$ in which aberration is not taken into account:

$$
\tilde{l}=\frac{l}{\left(1+z_{D}\right)^{2}}=\frac{L}{4 \pi \tilde{d}_{p}^{2}}, \quad \tilde{d}_{p}=\left(1+z_{D}\right) d_{p} .
$$

After inserting the definition of $d_{p}$, we get for the case of the closed model:

$$
\tilde{d}_{p}=\left(1+z_{D}\right)^{2} a_{0} \sin \chi,
$$


For the flat model $\sin \chi$ should be replaced by $\chi$, and for the open one - by $\sinh \chi$.

\section{Time delay cosmology}

\subsection{Basics of the model}

The cosmological effects of diffusion gravity considered above show that nontrivial changes can be expected in the cosmological models too. Here we consider the minimum necessary changes in the Friedmann model.

The solution of the doubling redshifts paradox showed that the first difference is the time delay during expansion. First of all, therefore, we will study the consequences of this change, i.e. consider the time delay cosmology.

From three models of constant curvature, open, flat and closed, we choose for definiteness the latter, closed. Only in it the volume of the universe is finite at every moment and therefore both the Big Bang and the conservation of the full energy of the background field in the whole space have a direct physical meaning.

The line element of the Friedmann closed model in the comoving frame, where the proper time $\tau$ plays the role of the time coordinate, has the form:

$$
d s^{2} \simeq c^{2} d \tau^{2}-a^{2}(\tau)\left(d \chi^{2}+S_{\chi}^{2} \cdot d \Omega_{(2)}^{2}\right),
$$

where $d \Omega_{(2)}^{2}=d \theta^{2}+\sin ^{2} \theta \cdot d \varphi^{2}$. In the time delay cosmology we will express $d \tau$ in terms of the present time $d t$ as $d \tau=d t \cdot a_{0} / a$ and (36) we rewrite in the form:

$$
d s^{2}=\frac{a_{0}^{2}}{a^{2}(t)} c^{2} d t^{2}-a^{2}(t)\left[d \chi^{2}+\sin ^{2} \chi\left(d \theta^{2}+\sin ^{2} \theta d \varphi^{2}\right)\right] .
$$

The time component of the metric and the determinant are, therefore, equal to

$$
g_{00}(t)=\frac{a_{0}^{2}}{a^{2}}, \quad \sqrt{-g}=a_{0} a^{2} \sin ^{2} \chi|\sin \theta| .
$$

The energy-momentum tensor of homogeneous dust with a local energy density $\rho$ is:

$$
T^{i k}=\rho \frac{d x^{i}}{d s} \frac{d x^{k}}{d s}, \quad T^{00}=\rho \frac{a^{2}}{a_{0}^{2}}, \quad T_{0}^{0}=T^{00} g_{00}=\rho, \quad T_{00}=\rho g_{00}=\rho \frac{a_{0}^{2}}{a^{2}}
$$

The energy-momentum conservation condition gives the relations ( $\dot{a}=d a / d t)$ :

$$
\begin{gathered}
\frac{1}{\sqrt{-g}} \frac{\partial}{\partial x^{k}}\left(T_{i}^{k} \sqrt{-g}\right)-\frac{1}{2} \frac{\partial g_{k l}}{\partial x^{i}} T^{k l}=0, \\
\frac{1}{\sqrt{-g}} \frac{\partial}{\partial t}\left(T_{0}^{0} \sqrt{-g}\right)-\frac{1}{2} \frac{\partial g_{00}}{\partial t} T^{00}=0, \\
\frac{1}{a^{2}} \frac{\partial}{\partial t}\left(\rho a^{2}\right)+\rho \frac{\dot{a}}{a}=0, \quad \frac{\partial}{\partial t}\left(\rho a^{3}\right)=0, \quad \rho a^{3}=\rho_{0} a_{0}^{3} .
\end{gathered}
$$

The Einstein's equations

$$
G_{i k}=\frac{8 \pi G}{c^{4}} T_{i k}
$$


with the Einstein tensor $G_{i k}=R_{i k}-R g_{i k} / 2$, where $R_{i k}$ is the Ricci tensor, $R=g^{i k} R_{i k}$, give:

$$
\frac{3}{a^{4}}\left(a^{2} \frac{\dot{a}^{2}}{c^{2}}+a_{0}^{2}\right)=\frac{8 \pi G}{c^{4}} \rho \frac{a_{0}^{2}}{a^{2}} .
$$

At simplifying by using (42):

$$
\frac{a^{2}}{a_{0}^{2}} \frac{\dot{a}^{2}}{c^{2}}+1=\frac{8 \pi G \rho a^{3}}{3 c^{4}} \frac{1}{a}=\frac{a_{m}}{a}, \quad a_{m} \equiv \frac{8 \pi G \rho_{0} a_{0}^{3}}{3 c^{4}},
$$

we get the evolution equation:

$$
\dot{a}^{2}=c^{2} \frac{a_{0}^{2}}{a^{2}}\left(\frac{a_{m}}{a}-1\right), \quad \dot{a}= \pm c \frac{a_{0}}{a} \sqrt{\frac{a_{m}}{a}-1} .
$$

As we see from the comparison with the Friedman equation:

$$
\left(\frac{d a}{d \tau}\right)^{2}=c^{2}\left(\frac{a_{m}}{a}-1\right), \frac{d a}{d \tau}= \pm c \sqrt{\frac{a_{m}}{a}-1},
$$

the expansion speed in (46) contains an additional factor $a_{0} / a$ showing a faster expansion speed in earlier epochs in terms of the time of our epoch. The light speed $\tilde{c}$ was also faster:

$$
\frac{a_{0}^{2}}{a^{2}} c^{2} d t^{2}-a^{2} d \chi^{2}=0, \quad \tilde{c}^{2} d t^{2}-a^{2} d \chi^{2}=0, \quad \tilde{c}=\frac{a_{0}}{a} c .
$$

For the modern epoch and the constant $H_{0}$ the Eq. (46) gives the usual expressions:

$$
\begin{gathered}
\dot{a}_{0}= \pm c \frac{\sqrt{1-a_{0} / a_{m}}}{\sqrt{a_{0} / a_{m}}}= \pm c \frac{\sqrt{1-b}}{\sqrt{b}}, \quad b \equiv \frac{a_{0}}{a_{m}}, \\
H_{0}=\frac{\dot{a}_{0}}{a_{0}}= \pm \frac{c}{a_{0}} \frac{\sqrt{1-b}}{\sqrt{b}} .
\end{gathered}
$$

For the time $t$ corresponding $a(t)$, the evolution equation (46) gives:

$$
\begin{aligned}
& c t=\frac{1}{a_{0}} \int_{0}^{a(t)} \frac{a^{3 / 2} d a}{\sqrt{a_{m}-a}}= \\
& =\frac{3 a_{m}}{4 a_{0}}\left[a_{m} \arcsin \left(\frac{2 a}{a_{m}}-1\right)-\frac{3 \pi a_{m}}{2}-\left(1+\frac{2 a}{3 a_{m}}\right) \sqrt{a\left(a_{m}-a\right)}\right]
\end{aligned}
$$

For proper time $\tau$, we obtain from (47):

$$
c \tau=\int_{0}^{a(t)} \frac{a^{1 / 2} d a}{\sqrt{a_{m}-a}}=\frac{a_{m}}{2} \arcsin \left(\frac{2 a}{a_{m}}-1\right)-\frac{3 \pi a_{m}}{4}-\sqrt{a\left(a_{m}-a\right)} .
$$

\subsection{The early universe}

The faster rate of proper times in earlier epochs explains a number of facts for which radical hypotheses had to be advanced before. In particular, if the scale factor was $10^{3}$ times 
smaller, then the light speed was just as fast $\tilde{c} \sim 10^{3} c$ in terms of our time, which explains the homogeneity and isotropy of $\mathrm{CMB}$ by a much faster mixing of the photon flux, which practically eliminates the horizon problem.

Moreover, since in a very early universe the particles of matter were ultrarelativistic, then they also moved several orders of magnitude faster than photons in our epoch, which also explains the large-scale homogeneity and isotropy of the whole matter.

Let's consider the changes in the cosmology of early eras shortly. The energymomentum tensor of ultrarelativistic matter and radiation with the local energy density $\rho$ and pressure $p=\rho / 3$, having the form:

$$
\begin{aligned}
& T^{i k}=\frac{d x^{i}}{d s} \frac{d x^{k}}{d s}(\rho+p)-p g^{i k}=\frac{4}{3} \rho \frac{d x^{i}}{d s} \frac{d x^{k}}{d s}-\frac{1}{3} \rho g^{i k}, \\
& T^{00}=\rho \frac{a^{2}}{a_{0}^{2}}, \quad T_{0}^{0}=T^{00} g_{00}=\rho, \quad T_{00}=\rho g_{00}=\rho \frac{a_{0}^{2}}{a^{2}}
\end{aligned}
$$

we insert into the energy-momentum conservation condition:

$$
\frac{1}{\sqrt{-g}} \frac{\partial}{\partial x^{0}}\left(T_{0}^{0} \sqrt{-g}\right)-\frac{1}{2} \frac{\partial g_{k l}}{\partial x^{0}} T^{k l}=0,
$$

and obtain the relations $(\dot{a}=d a / d t)$ :

$$
\frac{1}{a^{2}} \frac{\partial}{\partial t}\left(\rho a^{2}\right)+2 \rho \frac{\dot{a}}{a}=0, \quad \frac{\partial}{\partial t}\left(\rho a^{4}\right)=0, \quad \rho a^{4}=\rho_{0} a_{0}^{4} .
$$

The evolution equation (44) then transforms into:

$$
\frac{a^{2}}{a_{0}^{2}} \frac{\dot{a}^{2}}{c^{2}}+1=\frac{8 \pi G \rho a^{4}}{3 c^{4}} \frac{1}{a^{2}}=\frac{a_{m} a_{0}}{a^{2}},
$$

and:

$$
\dot{a}^{2}=c^{2} \frac{a_{0}^{2}}{a^{2}}\left(\frac{a_{m} a_{0}}{a^{2}}-1\right), \quad \dot{a}= \pm c \frac{a_{0}}{a} \sqrt{\frac{a_{m} a_{0}}{a^{2}}-1} .
$$

From a comparison with the Friedman equation:

$$
\left(\frac{d a}{d \tau}\right)^{2}=c^{2}\left(\frac{a_{m} a_{0}}{a^{2}}-1\right), \frac{d a}{d \tau}= \pm c \sqrt{\frac{a_{m} a_{0}}{a^{2}}-1},
$$

we see that the expansion speed contains an additional factor $a_{0} / a$ again, which leads to a faster expansion speed in the early epochs in terms of the time of our epoch.

For the time $t$ corresponding $a(t)$, the evolution equation (57) gives:

$$
c t=\frac{1}{a_{0}} \int_{a(t)}^{0} \frac{a^{2} d a}{\sqrt{a_{m} a_{0}-a^{2}}}=\frac{a}{2 a_{0}} \sqrt{a_{m} a_{0}-a^{2}}-\frac{a_{m}}{2} \arcsin \left(\frac{a}{\sqrt{a_{m} a_{0}}}\right) .
$$

For proper time $\tau$ we get: 


$$
c \tau=\int_{a(t)}^{0} \frac{a d a}{\sqrt{a_{m} a_{0}-a^{2}}}=\frac{1}{2} \sqrt{a_{m} a_{0}}\left(1-\sqrt{1-a^{2} / a_{m} a_{0}}\right) .
$$

\section{Observable effects of the time delay cosmology}

\subsection{The "distance modulus - redshift" relation}

For the world line of photons from distant sources we have:

$$
\frac{a_{0}^{2}}{a^{2}} c^{2} d t^{2}-a^{2} d \chi^{2}=0, \quad c d t= \pm \frac{a^{2}}{a_{0}} d \chi .
$$

Together with (46), this allows one to find the magnitude of the angle $\chi$ passed by the photon:

$$
\chi= \pm c \int_{t}^{t_{0}} \frac{a_{0}}{a^{2}} d t=c \int_{a}^{a_{0}} \frac{a_{0}}{a^{2}} \frac{1}{a_{0}} \frac{a^{3 / 2} d a}{\sqrt{a_{m}-a}}=c \int_{a}^{a_{0}} \frac{d a}{\sqrt{a\left(a_{m}-a\right)}}
$$

Integration gives:

$$
\sin \chi=\frac{c}{H_{0} a_{0}} \frac{a}{a_{0}} \cdot 2(1-b)\left[\frac{a_{0}}{a}-1+(1-2 b)\left(1-\sqrt{1+\frac{a_{0} / a-1}{1-b}}\right)\right] .
$$

For the extension of wavelengths, then there would be a relationship:

$$
\frac{a_{0}}{a}=1+z_{E}
$$

and from (63) the well-known Mattig's formula would follow. But in our case, the observed redshift $z$ is related to the Doppler effect as in (18) $z=z_{D}$, and then we calculate its contribution.

Unlike flat static space, where there was a simple recession law $v=H_{0} r$, in an expanding curved space, where the physical distance is equal to $\mathrm{r}(t, \chi)=a(t) \chi$, the situation is more complicated.

The local recession speed $\mathrm{v}(t, \chi)$ of objects depends on both distance and time, and there appears $\mathrm{H}(\tau)=a^{-1} d a / d \tau$ instead of $H_{0}$. Therefore, there is only a local law for the difference in the speeds $\delta \mathrm{v}$ of two close objects, the physical distance between which at the moment $t$ is equal $\delta \mathrm{r}$. From the definition of this distance

$$
\delta \mathrm{r}(t, \chi)=\mathrm{r}(t, \chi+\delta \chi)-\mathrm{r}(t, \chi)=a(t) \delta \chi
$$

we find the expansion speed of this spatial section in the form:

$$
\delta \mathrm{v}(t, \chi)=\frac{\partial}{\partial \tau} \delta \mathrm{r}(t, \chi)=\mathrm{H} \cdot a \delta \chi,
$$

which gives the desired local recession law:

$$
\delta \mathrm{v}(t, \chi)=\mathrm{H} \cdot \delta \mathrm{r}(t, \chi) .
$$

In relativistic cosmology, the rates of removal of fairly close objects on the hypersurface of simultaneity $t=$ const . are related by the relativistic relation: 


$$
\mathrm{v}(\chi+\delta \chi)=\frac{\mathrm{v}(\chi)+\mathrm{H} \delta \mathrm{r}}{1+\mathrm{v}(\chi) \cdot \mathrm{H} \delta \mathrm{r} / c^{2}}
$$

which, due to smallness of $\delta \mathrm{v} / c$, can be written as

$$
\mathrm{v}(\chi+\delta \chi)-\mathrm{v}(\chi) \simeq\left[1-\mathrm{v}^{2}(\chi) / c^{2}\right] \mathrm{H} a \cdot \delta \chi
$$

From this we obtain the differential equation for determining the velocities:

$$
\mathrm{H} a \cdot d \chi=\frac{d \mathrm{v}}{1-\mathrm{v}^{2} / c^{2}},
$$

the solution of which under the condition $t=$ const. gives the dependence of the removal rate on the physical distance:

$$
\mathrm{r}(t) \simeq \frac{c}{\mathrm{H}} \int_{0}^{\mathrm{v}(t, x)} \frac{d(\mathrm{v} / c)}{1-\mathrm{v}^{2} / c^{2}} \mathrm{r}=\frac{c}{\mathrm{H}} \operatorname{arcth}(\mathrm{v} / c)=\frac{c}{\mathrm{H}} \ln \sqrt{\frac{1+\mathrm{v} / c}{1-\mathrm{v} / c}} .
$$

As we see, the expression under the logarithm is the redshift factor $\left(1+z_{D}\right)$ in the longitudinal Doppler effect. This gives a simple formula expressing $\mathrm{r}$ in terms of $z$ :

$$
\mathrm{Hr}=c \ln \left(1+z_{D}\right) .
$$

Поскольку Н пропорционален $H_{0}$ и зависит от фактора $a_{0} / a=\left(1+z_{E}\right)$, то:

$$
\begin{gathered}
\frac{\mathrm{Hr}}{H_{0} \mathrm{r}_{0}}=\frac{\dot{a}}{\dot{a}_{0}} \frac{a_{0}}{a} \frac{\mathrm{r}}{\mathrm{r}_{0}}=\frac{\dot{a}}{\dot{a}_{0}}=f, \quad \mathrm{Hr}=H_{0} \mathrm{r}_{0} f, \\
f\left(z_{E}, b\right)=\sqrt{\frac{a_{0} / a-b}{1-b}}=\sqrt{1+\frac{z_{E}}{1-b}}
\end{gathered}
$$

Next, we make an approximation

$$
f \mathrm{r}_{0}=\mathrm{r}_{0} \sqrt{1+\frac{z_{E}}{1-b}} \simeq r_{0} \cdot\left(1+z_{E}\right)
$$

the validity of which will be evaluated later. Substituting this into (72) we obtain:

$$
r\left(z_{D}, z_{E}\right) \simeq \frac{c}{H_{0}} \frac{\ln \left(1+z_{D}\right)}{1+z_{E}} .
$$

At small distances $z_{D}, z_{E} \ll 1$, and the formula (76) passes into the linear law:

$$
\mathrm{v} \simeq H_{0} r \simeq c z_{D} .
$$

The apparent and absolute luminosities of sources $l, L$ are related to the photometric distance $d_{p}$ as $l=L / 4 \pi d_{p}^{2}$. Through apparent and absolute magnitudes, $m$ and $M$ they are expressed as $\quad l=10^{-m / 2.5} \cdot 2.52 \cdot 10^{-5} \mathrm{erg} / \mathrm{cm}^{2} \mathrm{sec}$, $L=10^{-M / 2.5} \cdot 3.02 \cdot 10^{35} \mathrm{erg} / \mathrm{cm}^{2} \mathrm{sec}$. Expansion leads to a decrease in the energy and frequency of arrival of photons by a total value $\left(1+z_{D}\right)^{2}$, and relativistic aberration reduces 


\section{Cosmology of diffusion gravity}

the apparent luminosity by the same factor $\left(1+z_{D}\right)^{2}$. As a result, for apparent luminosity we obtain the expression:

$$
l_{F}=\frac{L}{4 \pi d_{p}^{2}}=\frac{L}{4 \pi r^{2}} \cdot \frac{1}{\left(1+z_{D}\right)^{4}} .
$$

The photometric distance $d_{p}$ is thus equal to:

$$
d_{p, 0}=\left(1+z_{D}\right)^{2} r=10^{-5+(m-M) / 5} M n c,
$$

from which for the distance modulus $\mu \equiv m-M=5 \lg \left(d_{p}\right)+25$, after substitution (76) in (80), we obtain the «distance modulus - redshift" relation $\left(A \equiv 5 \cdot \lg \left(c / H_{0}\right)+25\right)$ :

$$
\mu=5 \lg \left[\left(1+z_{D}\right)^{2} r\right]+25=5 \lg \left[\frac{\left(1+z_{D}\right)^{2}}{1+z_{E}} \ln \left(1+z_{D}\right)\right]+A
$$

The value of $1+z_{E}$ is proportional to the distance, and hence the recession speed of the object, while $1+z_{D}$ at large distances it also contains a quadratic dependence on speed. Thus, $z_{D}$ is larger $z_{E}$ and the difference is in a quadratic part. The relationship between them at close distances $v \ll c$ has the form $(\beta=v / c)$ :

$$
z_{E} \simeq \beta, \quad z_{D} \simeq \beta+\frac{1}{2} \beta^{2}, \quad 1+z_{E} \simeq \sqrt{1+2 z_{D}} \simeq 1+z_{D}-\frac{1}{2} z_{D}^{2}
$$

For estimates, in the general case, we express $z_{E}$ through $z_{D}$ using a small parameter $\varepsilon$ :

$$
1+z_{E} \simeq\left(1+z_{D}\right)\left(1-\varepsilon z_{D}\right), \quad \varepsilon \ll 1 .
$$

In such a "basic" representation (80) is simplified and takes the form:

$$
\mu=5 \lg \left[\frac{1+z_{D}}{1-\varepsilon z_{D}} \ln \left(1+z_{D}\right)\right]+A .
$$

In Fig. Figure 1 compares (80) with observational data on Type $1 a$ supernovae, where $H_{0}=0.70 h$ is taken from the linear part. As we see, the theoretical curve is consistent with the observations at $\varepsilon \simeq 0.08$. Further refinement of the approximations and data processing will give even more realistic curves. 


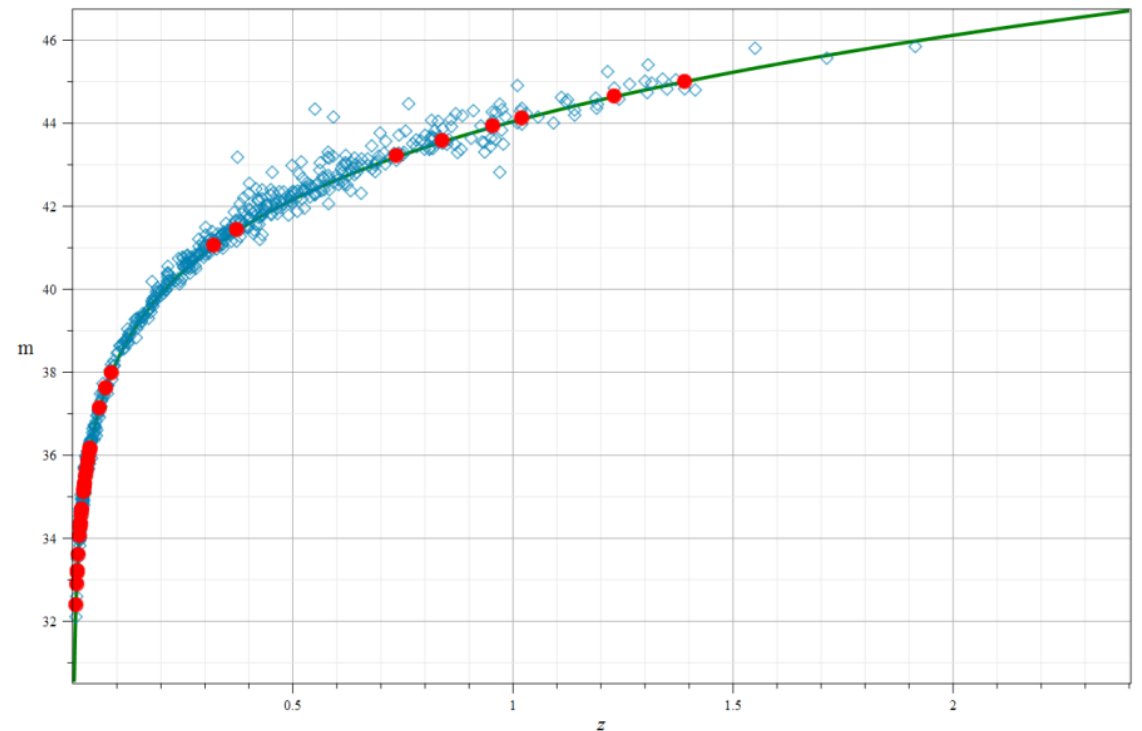

a)

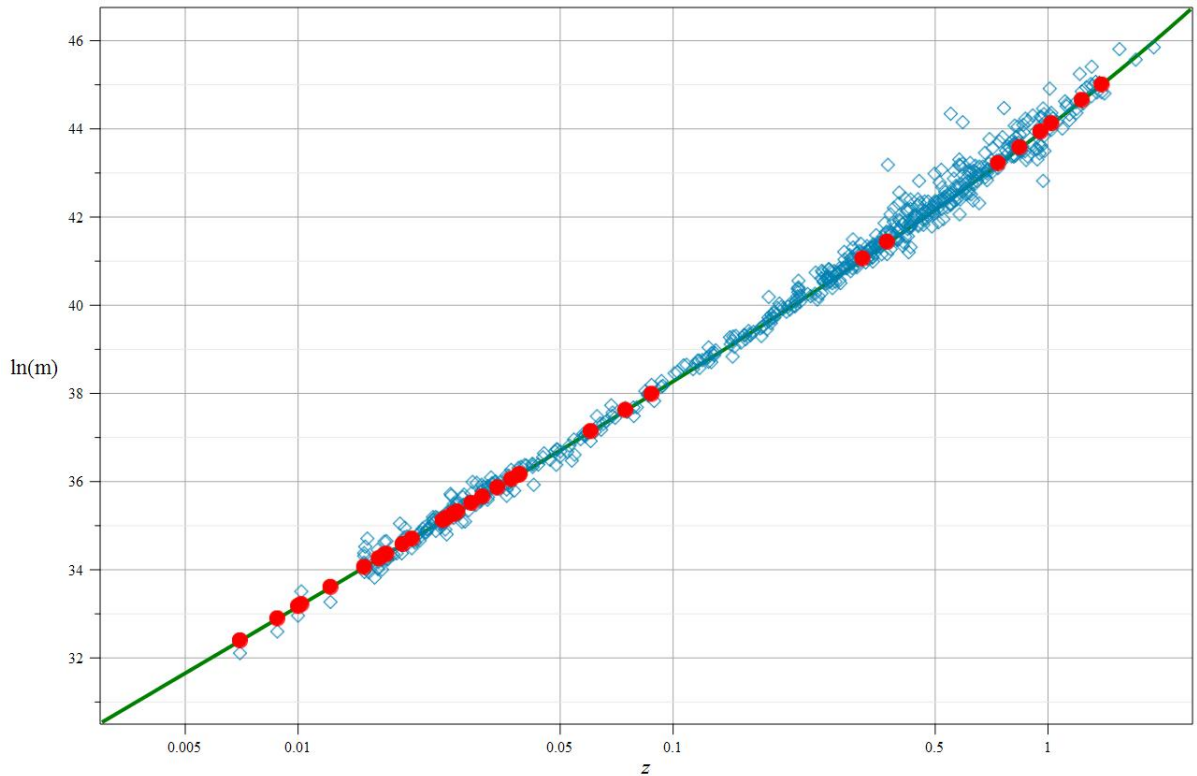

b)

Fig. 1. The plots "apparent magnitude - redshift" for supernova 1a (green curve), a) in the usual and b) in the logarithmic scale. The data include about $600 \mathrm{SN} 1 \mathrm{a}$ from several collections (blue dots) and 33 "pure" supernovae 1a from [6] (red dots).

\subsection{Changes in the properties of $\mathrm{CMB}$}

The observed CMB, which emitted in a very early universe, allows one to study closer epochs, in particular, the recombination period, when the radiation disconnected from matter. Notice some changes in the description of the relict background, which follow from the main distinctions of the new treatment from the previous one.

The ratio of the temperature of the CMB in an earlier epoch $T_{e}$ to its observed temperature $T_{r}$ is determined by the ratio of the observed wavelength $\lambda_{r}$ to the wavelength in 
that epoch $\lambda_{e}$. In the Friedmann model, the redshift was attributed to stretching and therefore the ratio of wavelengths was given by the ratio of scale factors $a_{0} / a$, and therefore depended on $z_{E}$, and also provided an equation for $T(a)$ :

$$
\frac{T_{e}}{T_{r}}=\frac{\lambda_{r}}{\lambda_{e}}=\frac{a_{0}}{a}=1+z_{E}, \quad \frac{d T}{T}=-\frac{d a}{a} .
$$

In the new model, all the redshifts of the radiation are determined by the Doppler effect and therefore the temperature ratio now depends on $v(a)$ and $z_{D}$ :

$$
\frac{T_{e}}{T_{r}}=\frac{\lambda_{r}}{\lambda_{e}}=\sqrt{\frac{1+v / c}{1-v / c}}=1+z_{D} .
$$

As a result, the relation between redshifts $z_{D}$ and $a$ now turns out to be more complicated, as it must be specified $v(a)$. But the fact that $z_{D}$ appears instead of $z_{E}$ already reveals a number of significant changes.

Unlike linear stretching of wavelengths, the relativistic Doppler effect has a quadratic part (see (19)), which dominates at large $z_{D}$, which means that the relict background is almost completely described by this part of the effect. With the same $a$, we have $z_{D}>z_{E}$, and therefore, re-treatment of the measured redshift as a Doppler shift $z_{E} \rightarrow z_{D}$ without changing its value means that it now refers to much larger $a$ than previously thought, i.e. to a much later time $t$.

Larger values $a$ and later times $t$ mean significantly lower densities of matter and radiation in that epoch when the relict background detached from matter. But at $z_{D} \sim 1500$ gives $v \simeq c-\delta v, \delta v \ll c$, and (85) takes the form:

$$
\frac{T_{e}}{T_{r}}=\frac{\lambda_{r}}{\lambda_{e}} \simeq \sqrt{\frac{2 c}{\delta v}} \simeq z_{D}, \quad \frac{\delta v}{c} \simeq \frac{2}{z_{D}^{2}} \simeq 9 \cdot 10^{-7}
$$

and heterogeneities in the recombination epoch, through which the relict stream passed, would look flattened for us due to relativistic contraction.

Despite a later time and a larger scale factor than in the Friedmann model, processes in the recombination epoch went faster, and temperatures were higher by a factor $a_{0} / a=1+z_{E}$

And, finally, another important change concerns measurements of the characteristics of the relict background in our time, from which the value is determined [7]:

$$
\Omega_{m} h_{0}^{3} \simeq 0.096 .
$$

From this value, assuming $\Omega_{m} \simeq 0.315$, then a value $h \simeq 0.67$ was fixed, which was significantly different from $h \simeq 0.73$ determined from redshifts and apparent luminosities of Type 1a supernovae. In the new model, on the contrary, the supernova redshifts fix the value $h \simeq 0.70$ (or $h \simeq 0.73$ ), and then $\Omega_{m}$ can be determined from the CMB data by using the empirical relation (87), which gives $\Omega_{m} \simeq 0.28$ (or $\Omega_{m} \simeq 0.25$ ). These lower values are confirmed by other independent observations of the distribution of galaxies that give $\Omega_{m} \simeq 0.26$. Thus, in the new model there is no contradiction between the supernova data and the CMB observation, on the contrary, they only complement each other. 


\subsection{The lack of the former cosmological problems in the model}

a. The lack of flatness problem and not need in fine tuning

In the Friedmann model, in early periods, space becomes flat with high accuracy. Indeed, we write the evolution equation (58) in the general case of a flat, closed and open models with $k=0, \pm 1$ as:

$$
\rho_{c} \frac{a^{2}}{a_{0}^{2}}=\rho-k \frac{A}{a^{2}}, \quad \rho_{c}=\frac{3 c^{2} H^{2}}{8 \pi G}, \quad A=\frac{3 c^{4}}{8 \pi G}
$$

or through the curvature parameter $\Omega=\rho / \rho_{c}$ as:

$$
\Omega^{-1}-1=-\frac{k A}{\rho a^{2}} \text {. }
$$

Then, at the dominance of matter or radiation, we have:

$$
\begin{aligned}
& \frac{\Omega_{0}^{-1}-1}{\Omega^{-1}-1}=\frac{\rho a^{3}}{\rho_{0} a_{0}^{3}} \frac{a_{0}}{a}=\frac{a_{0}}{a}, \quad \rho a^{3}=\text { const. } \\
& \frac{\Omega_{0}^{-1}-1}{\Omega^{-1}-1}=\frac{\rho a^{4}}{\rho_{0} a_{0}^{4}} \frac{a_{0}^{2}}{a^{2}}=\frac{a_{0}^{2}}{a^{2}}, \quad \rho a^{4}=\text { const. }
\end{aligned}
$$

and in both cases $\Omega$ at $a \rightarrow 0$ tends to a flat value $\Omega \rightarrow 1$, i.e. to $k=0$.

In the time delay cosmology, there is no flatness problem, since the curvature parameter $\Omega$ decreases in early epochs. The evolution equation (57), which in the general case $k=0, \pm 1$ has the form:

$$
\rho_{c} \frac{a^{2}}{a_{0}^{2}}=\rho-k \frac{A}{a^{2}},
$$

we can represent in the form similar to (89):

$$
\frac{a^{2}}{\Omega a_{0}^{2}}-1=-\frac{k A}{\rho a^{2}} \text {. }
$$

Now we have:

$$
\begin{aligned}
& \frac{\Omega_{0}^{-1}-1}{a^{2} /\left(\Omega a_{0}^{2}\right)-1}=\frac{a_{0}}{a} \frac{\rho a^{3}}{\rho a_{0}^{3}}=\frac{a_{0}}{a}, \\
& \frac{\Omega_{0}^{-1}-1}{a^{2} /\left(\Omega a_{0}^{2}\right)-1}=\frac{a_{0}^{2}}{a^{2}} \frac{\rho a^{4}}{\rho a_{0}^{4}}=\frac{a_{0}^{2}}{a^{2}} .
\end{aligned}
$$

This shows that in both cases at $a \rightarrow 0$ the parameter $\Omega$ tends to zero as $a^{2}$ :

$$
\Omega=\frac{a^{2}}{a_{0}^{2}} \cdot \frac{1}{1+\left(\Omega_{0}^{-1}-1\right) a / a_{0}} \rightarrow \frac{a^{2}}{a_{0}^{2}} .
$$

Thus, in terms of the time of our epoch, there is no flatness problem and there is no need for fine tuning.

\section{b. Homogeneity and isotropy as a result of faster evolution}




\section{Cosmology of diffusion gravity}

Similar to the spherical star models, the large-scale homogeneity and isotropy of the distribution of matter in the model are consequences of a causal relationship between different regions under conditions of faster light speed and faster evolution.

During the radiation-dominated and recombination epochs, particles of matter diffuse as a small admixture in a high-temperature gas of photons, and such diffusion smooth out significant inhomogeneities in the distribution of matter and radiation in causally related regions with the large values of the light speed in those epochs.

\section{c. The lack of horizon problem}

The horizon problem of the Friedmann model was that, on the one hand, at the expansion the size of a causally related region (horizon) grows as $r_{h o r} \approx t$, while the scale factor grows more slowly - as $a \approx t^{1 / 2}$ in early epochs and as $a \approx t^{2 / 3}$ in later epochs. On the other hand, it was believed that the CMB flux ceased to interact with matter after the recombination epoch and the radiation density in causally unrelated regions could not be aligned in any way, but in fact, isotropic and homogeneous fluxes come to us from them on average.

In the new model, the curvature radius of the universe and the size of the causally related region grew much faster in early epochs than was assumed in the Friedmann model. The light speed was also faster in terms of the time of our epoch. Thus, in this model there is no horizon problem.

\section{d. The lack of the initial singularity problem}

In the former standard cosmology, it was still considered the contraction of the entire

universe up to the Planck volume $V_{p l} \sim l_{g}^{3}$. However, each of the individual elementary particles is an "external observer" for any other particle. As a result, at approaching to $l_{g}$ the proper time of the particles, they are slowed down relative to the world time of the center of inertia of this system of particles.

Therefore, in fact, in GR it is necessary to consider the compression of each of the $N_{0}$ current particles and the $\Delta N$ creating quanta to the volume $V_{p l}$, after which the wavelength of these particles ceases to decrease. As a result, the minimal volume of the universe $V_{\min }$, to which, in principle, compression can be continued, is the sum of these elementary volumes of each of the particles $V_{p l}$ and in units of the Planck volume it will always be greater than the current number of particles in the universe:

$$
V_{\min }>N_{0} V_{p l} \sim 10^{88} V_{p l} .
$$

When this smallest volume $V_{\text {min }}$ is reached, the particles will become frozen due to their own strong gravitational fields and the compression of the universe will stop in terms of cosmological proper time $\tau$ too. This restriction from GR has a fundamental and modelindependent character, since it follows from the classical (non-quantized) component of the gravitational field.

e. The lack of entropy problem 
The problem of a large value of entropy also arose earlier due to the fact that the universe was supposed to be compressed to the one volume $V_{p l}$.

In the new model, compression continues only to the minimal volume $V_{\min }$, while maintaining the number of particles no less than the current one, and there is no problem of a large entropy. In this model, the total energy of the universe is non-zero and is conserved. A large number of elementary volumes at ultimate compression and the corresponding "number of cells" characterize the entropy of the universe, which is further preserved as well as the residual average number of particles is preserved in later periods of expansion.

\section{f. The lack of a cosmological constant problem}

The former widely accepted cosmological paradigm was mainly based on the introduction of the cosmological constant $\Lambda$. However, its value turned out to be so small that it could not be explained not only by the Standard Model of particle physics, but also by its hypothetical generalizations. This is the cosmological constant problem, which turned out to be practically insolvable in the framework of the former standard paradigms in both cosmology and particle physics.

In reality, particle physics provides neither theoretical nor observational grounds for introducing a zero-point vacuum energy [5]. This means that cosmology can be in agreement with particle physics only in the absence of the cosmological constant $\Lambda=0$.

In the new model the cosmological constant is absent, and Einstein's equations with matter energy-momentum density only are quite sufficient to describe the observations. This means reaching agreement between cosmology and particle physics in the problem of vacuum energy.

\section{g. The lack of the cosmological dark matter problem}

Cosmological models set limits on the density of non-baryonic dark matter. In a model with a slowing time, there is no such need, although in principle a slight admixture of such matter is not excluded.

In any case, both the practical absence and the presence of some small admixture of dark matter is not a problem, and therefore there is no problem of dark matter in the new model.

\section{h. The charge asymmetry as an initial condition}

The absence of antiparticles (positrons, antiprotons and antineutrons) in the observed universe formally follows from the fact that $\mathrm{CP}$ symmetry is violated in the hot phase, which leads to an excess of the number of particles over antiparticles of order $10^{-8} \div 10^{-9}$, which is enough to form the current ratio of the numbers of fermions and photons.

In the previous approaches, they were limited mainly to mechanisms of violation of baryon charge conservation within the framework of hypothetical models of the Grand Unification, then in this interpretation, composite models in which charge symmetry can be restored at the level of subparticles

Unlike previous approaches, in the new cosmology the composite models, in which symmetry between particles and antiparticles can be restored at the level of subparticles, look preferable. 


\section{Conclusion}

Thus, the Friedmann's model of cosmology used so far, even with the inclusion of dark matter and dark energy, has led to the redshifts doubling paradox due to the necessity to take into account both the Doppler effect and the stretching of photon's wavelengths at the space expansion. This paradox meant the contradiction of the Friedman model with observations, and therefore the standard model of relativistic cosmology, based on it, was in fact inconsistent.

However, this paradox becomes naturally solved if one takes into account that the cosmological principle actually requires the same local frequencies of photons at different places only at the same epoch. And in different epochs, the energy levels of atoms, emitting photons, can differ even in the same place if the local proper times either slow down or speed up during expansion, which is fully compatible by GR.

Of these two possibilities, diffusion gravity unambiguously selects the first one, since the energy density of the background field decreases during expansion, which reduces the intensity of quantum fluctuations, and hence the rate of local proper times. The cosmological model formulated in the paper with slowing down local proper times naturally solves the doubling redshifts paradox. In this model, in earlier epochs, photons are emitted with a violetshift, which is compensated during the propagation of the photon by a redshift due to stretching. As a result, the photon wavelengths at registration contain only the contribution of the relativistic Doppler effect.

But if there is a relativistic Doppler effect, there is a relativistic aberration also, which leads to additional diminishing of distant sources due to a decrease in the number of photons per unit solid angle. Therefore, the new model differs from the Friedmann model also by taking into account relativistic aberration.

The new "distance modulus - redshift" relation is consistent with data on Type 1a supernovae. A new, more complicated dependence of redshifts on speed and scale factor removes disagreement on the values of $H_{o}$ from the CMB and supernovae data.

The new model leads to a number of non-trivial consequences for the early universe and allows one to naturally solve the well-known cosmological problems appeared in the Friedmann model.

A more details of cosmology with a slowing down proper times following from diffusion gravity are presented in the book [2].

\section{References}

1. Zakir, Z. (2020) Quant. and Grav. Phys., 1:003-7129; 1:004-7129.

2. Zakir, Z. (2020) Diffusion Quantum Mechanics and Diffusion Gravity. CTPA. T.

3. Zakir, Z. (2014) TPAC, 9, 75.

4. Hofmeister A. M., Criss R. E. (2020) Galaxies. 8, 47.

5. Zakir, Z. (2020) Quant. and Grav. Phys., 1:001-7128; 1:002-7128.

6. Pruzhinskaya M. V. et al. (2011) Astr. L. 37, 663.

7. Planck 2018 results. VI. Cosmological parameters. arXiv:1807.06209.

8. Zakir, Z. (2020) Quant. and Grav. Phys., 1:006-7130; 1:007-7130. 